\title{
A resilient multi-path routing for the SDN based space- based network
}

\author{
Feihu Dong ${ }^{1}$, Xi Chen ${ }^{2}$, Yasheng Zhang ${ }^{3}$, Zhongyuan Jiang ${ }^{4}, \mathrm{Ci} \mathrm{He}^{5}$ \\ \{6891hfd@163.com¹, chenqian1997@stu.xidian.edu.cn², zys163@163.com³ \\ zyjiang@xidian.edu.cn ${ }^{4}$, heci1027@ sina.com ${ }^{5}$ \}
}

Beijing University of Posts and Telecommunications, Beijing 100876, China ${ }^{1}$; Xidian University, Xi'an, Shaanxi 710071, China ${ }^{2,4}$; Science and Technology on Communication Networks Laboratory, Shijiazhuang, Hebei 050081, China ${ }^{1,2,3,4}$; The 54th Research Institute of CETC, Shijiazhuang, Hebei 050081, China $^{5}$

\begin{abstract}
To adapt the development of global communication and the rapid upgradation of communication technology(e.g. $4 \mathrm{G}$ to $5 \mathrm{G}$ ), the space-based network (SBN) is regarded as a very important networking architecture to improve the efficiency of data communication. However, with the increasing need of bandwidth, the SBN faces many open challenges: 1) high cost of update and deployment and complex implementations; 2) low robustness or resilience against the dynamic network structure changes. In this work, we first incorporate the SDN (Software Defined Network) architecture into the SBN to ensure the feasible implementation by separating the control panel and the data panel. Secondly, we present a new multi-path routing (MPR) to adapt the fast network state change and enhance the network resilience. Finally, we compose extensive experiments on both random networks and scale-free networks to verify the effectiveness of our MPR mechanism which has potential applications in the future deployment of SBN.
\end{abstract}

Keywords: Multi-path routing, SDN, Space-based network, Resilience.

\section{Introduction}

With the rapid generation upgrading of communication technology (e.g. $4 \mathrm{G}$ to $5 \mathrm{G}$ ), the bandwidth needs are enlarging, and the user applications are enriching. It requires the larger bandwidth and more timely communication. Moreover, data communication is in urgent need of lots of wide areas (e.g. the remote oceans and depopulated zones) which are not covered by the data network yet. In other words, it needs to achieve global communication in the next or future generation of communication technologies. However, the traditional communication network cannot deploy base stations to some harsh environment such as desert and ocean. Therefore a lot of research work concentrates on the space-based network(SBN) which can be 
deployed into the space to achieve full signal coverage and provide data communication service.

However, there are three characters in the SBN: (1) the SBN topology is dynamic because of the high speed of satellite nodes; (2) the space-based network is cyclical; (3) the spacebased system is vulnerable to interference and damage. Therefore a lot of research concentrate on the network routing algorithm to increase the robustness, resilience of data transmission in the space-based network [1-2].

In view of the problems such as the difficulty of the satellite topology changes frequently and the difficulties in network management caused by the characters of space-based network nodes and network deployment, software defined network (SDN) is utilized to solve the problem. In our research, we adopt the architecture of SDN to improve communications efficiency of the space-based network.

Concerned about the resilience and robustness of communication, there are a lot of routing algorithm is proposed. In the past decade, many researchers concentrate on the satellite networks routing. Lu et al. [3] propose a novel inter-satellite routing based on link recognizing to improve the network routing problem and reduce the packet loss and delay. $\mathrm{Li}$ et al.[4] propose a novel routing strategy based on fuzzy theory for satellite network to evaluate the satellite congestion and then decrease the network congestion and packet loss. Liu et al.[5] analyze the efficiency of routing algorithms for satellite network under a single satellite node fault and random node fault. Wang et al.[6] propose two-layer compressed search algorithm which is a routing search algorithm in satellite IP switches. Feng et al.[7] designs a intersatellite routing based on Fibonacci Heap to improve the routing efficiency of inter-satellite. $\mathrm{Xu}$ et al.[7-8] impose the integrated routing algorithm for multi-satellites, multi-groundstations and multi-processing-centers which improves routing performance of the satellite and ground station. Considering about the topology of SBN is dynamic. Some packet could be lost in the process of data transmission because of the topology changes. So it is necessary to design the efficient routing to achieve the robustness in the process of data transmission [9-13]. However, some innovative routing algorithms lead to networking performance degradation such as increasing the average path length [14-17] and the transmission time and even leading the network congestion. Take into account these research, we propose a novel routing algorithm.

In this work, we introduce an innovative multi-path routing algorithm to improve the space-based network performance. In the paper, the multi-path routing mechanism is proposed, the resilience of a space-based network is studied when a certain proportion of links are destroyed and a large number of delay verification experiments is done.

\section{SDN based Space-based Network (SBN)}

In general, the framework of SDN separates the underlying infrastructure from application programs and network services, makes network control directly 
programmable, and only forwards data according to the control information of the control layer. Therefore the SDN is cost-efficient, dynamic and controllable. But due to the deployment cost of space-based network is high. Therefore the combination of spacebased network and SDN increases network efficiency and scalability and reduces the overhead on the satellite. And space-based network based SDN is easy to manage and the utilization rate of network resources is high [18].

As the above shows, the framework of SDN is adapted to the satellite network. Firstly, the operation of the satellite network is periodic and regular. Therefore the controller can make full use of these information to compute and manage the network. Secondly, satellites move fast and the topology is dynamic. The SDN controller performs real-time according to the topology information, so as to make the network run efficiently and increase the network stability. Thirdly, the deployment cost of the satellite is expensive, so updating and reconfiguring the system of satellite is difficult. SDN architecture could reduce the expenditure.

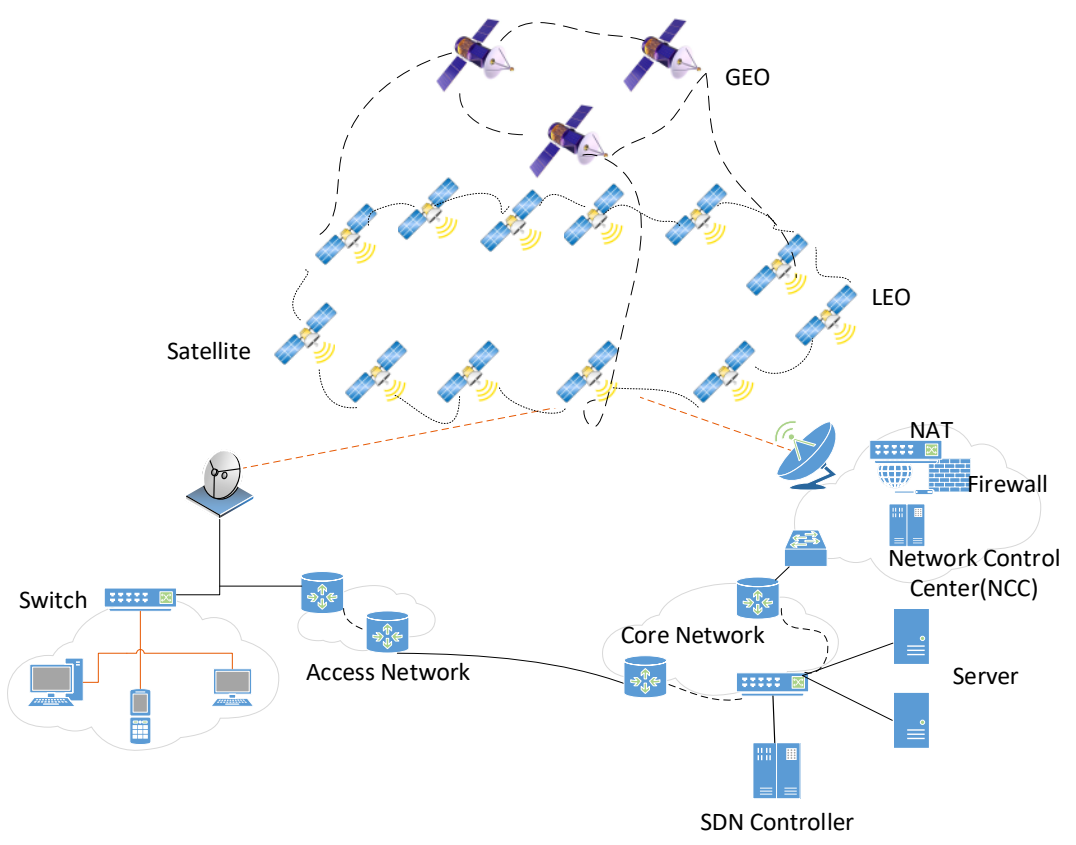

Fig. 1. The architecture of SDN-based SBN network.

As the Fig.1 shows, the SBN consists of low earth orbit(LEO) satellite and geosynchronous Earth orbit(GEO) satellite. SDN divides the network into two layers: control layer and data layer. Part of ground and GEO satellite is belonging to the control layer. The data layer consists of relay nodes on the ground and LEO satellites which transmit the information of the flow table. The control layer is responsible for the calculation and management of the network. The control layer has the information and 
traffic distribution of all communication nodes in the network, so it can manage the whole network more flexibly. The data layer just needs to transmit the packet.

Therefore we deploy the time-varying topology on the control layer and when switch over time slice, the control layer update route of the data layer. In the paper, we study an innovative routing algorithm and deploy it on the control layer. Then the data packet of data layer follow the rule to transmit and the control layer collect the information of all the satellite in the data layer.

\section{The Multi-path Routing (MPR)}

In the future of SBN deployment, the routing robustness is still a key problem to achieve the communication immediacy and security in the global communication network[13]. In the paper, we propose a Multi-path Routing (MPR) to compute multiple paths for every node pair and enhance the routing resilience against the changing network topology. The process is 1) we compute multiple paths for every node pair; 2) the first path is used for the data communication; 3) when the first path is unusable, we randomly select one available path from the alternative paths. In case of network failure, one of the multiple paths will be used as standby path and the communication will be restored.

Here we propose a novel multi-path routing with the following calculation flow:

(1) Given a network topology, path set $P$ and the link between node $i$ and $j$ in the topology has a weight $w_{i j}$ which is equal to 1 or the reciprocal of bandwidth or distance.

(2) Computing a path from source node to destination node with the lowest weight by Dijkstra algorithm [16].

(3) To judge whether the path already exists in the path set $P$, and eliminate duplicate paths.

(4) Adding an increment $\Delta w(\Delta w=1)$ to each link weight $W$ on the calculated path and updating the $W$.

(5) Calculating single path repeatedly until $k$ ( $k$ is an integer) paths are obtained.

For example we compute the multi-path from node 1 to node 6 in the Figure.2:(1) the weight of links which connect two reliable nodes is 1 , the weight of links which connect one reliable node is 2 , the other is 3 . And the path set between node 1 and node 6 is empty (2) we compute the path which weight is smallest: $\{1,2,4,6\},\{1,2,5,6\}$ and $\{1,3,5,6\}$ (the smallest weight is $3:\{1,2,4,6\})$, (3) judge the path $\{1,2,4,6\}$ is not in the path set. (4) add a $\Delta w=1$ to the weight of the link $(1,2),(2,4)$ and $(4,6)$, update the weight $w(1,2)=w(2,4)=w(4,6)=2$. $(5)$ calculate the second path $w\{1,2,5,6\}=2+2+2=6, w\{1,3,5,6\}=2+3+2=7$, the $w\{1,2,5,6\}$ is smaller and joins in a path set, then update the weight. And then continues the step (2)-(4). Finally we compute three path weight $w\{1,2,4,6\}=3, w\{1,2,5,6\}=6, w\{1,3,5,6\}=8$. When we 
choose a path from source to destination, we will choose the path which the weight is smallest to reduce the rate of loss packets and increase the stability of the network.

As the Fig.2 shows that different algorithm get available paths between source node and destination node compared with MPR, shortest path routing(SPR), node disjoint multi-path $S P R$ (NDMP-SPR), efficient routing (ER) [10] and node disjoint multi-path ER(NDMP-ER). And the number of paths of MPR is more than the other three algorithms.

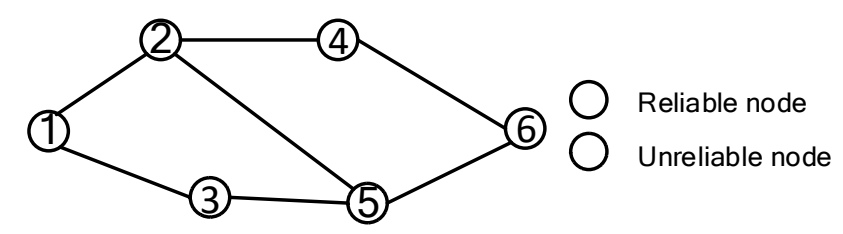

\begin{tabular}{|c|c|c|c|c|c|}
\hline- & SPR & NDMP-SPR & ER & NDMP-ER & MPR \\
\hline Reliable path & & & & $1-2-4-6$ & $1-2-4-6$ \\
\hline Unreliable path & $1-2-5-6$ & $1-2-5-6$ & $1-3-5-6$ & $1-3-5-6$ & $\begin{array}{c}1-2-5-6 \\
1-3-5-6\end{array}$ \\
\hline
\end{tabular}

Fig.2. The application and comparison of multi-path routing

In the network, there are always some unreliable nodes which are vulnerable to attack or link failure as shownn in Fig.2. The multi-path routing we designed is a routing algorithm with increasing weight, which can find out $k$ different paths for data transmission to avoid unreliable nodes and links and improve communication security and resilience.

\section{The Resilience of SBN}

According to the periodic changes of LEO satellite network topology, the SBN will calculate the network topology information of each time slice. When the time slice is switched, the LEO satellite will call the routing information of the corresponding time slice and transmit the packet according to the routing table, and adopt multi-path backup to forward the packet in case of failure. In the process of time slice switching, the link failure occurs when the link corresponding to the previous time slice does not exist on the current time slice. Check whether the multi-path information of the satellite node can reach. The multi-path routing ensures the stability of data transmission and reduces packet loss during time slice switching. In the case of multi-node and multi-link failure, the network ensures the immediacy of spacebased network communication and the robustness of network performance.

There are three cases of space-based network failure: (1) single node failure: if the time slice is not switched, but the node fails, we will find available path which weight is smallest from the backup path to transmit the packet; (2) time slice switching: if the time slice is switched to $T$. We will choose a route which can reach the destination node from the backup paths; (3) multi-node failure: if there is a multi-node failure in time slice $T$, the node will find 
an available path from its multiple backup paths, then based on the acyclic and forced forwarding, if the node has no path available and the data packet will timeout retransmission.

Here we test the resilience of SBN in different routing algorithms on the successful forwarding rate and the average path length of maximal connected subgraphs. In this experiment, we use the network of 200 satellite nodes to do the elastic experiment of spacebased network. When the failure rate is upto $20 \%$, the multi-path routing technology can recover the data forwarding of the route, and in the failure caused by the single node, multinode and time slice switching, achieve the immediacy of the communication within $50 \mathrm{~ms}$.

In the novel of MPR, we set the node failure ratio as $f$, and the path budget in the multipath routing algorithm as $k$ which is the maximum value of a path set.

\section{Experiments and results}

As we known, the traffic efficiency is related to the successful forwarding rate and average path length. Therefore, we test these parameters in the paper. Here we focus on the resilience of the network in one time slice because of the destruction of nodes and links in the BA network and ER network. The network scale is 200, and the average degree is 4 . The result is that the average value of 10 networks with the same network size.

\subsection{Network model}

Here we adopt two kinds of network models in the experiment: 1) the ER network model, 2) the BA network model.

ER network, one kind of random network, starts with $N$ nodes and connects each pair of nodes with probability $p$, which builds a graph with approximately $p N(N-1) / 2$ randomly placed links. Node degree of ER network follows a Poisson distribution, which indicates that most nodes have approximately the same number of links. In the recent research, most simulation adopt the ER network to simulate the network architecture [19].

BA network is one kind of scale-free networks, which is characterized by a power-law degree distribution, the probability that a node has $k$ links follows $P(k) \sim k^{-\gamma}$, where $\gamma$ is the degree exponent. A new node tends to connect to an old node of high degrees in the BA network which conforms to the law of real network. Same as the ER network, the architecture of the BA network is used to be simulated in some network research [20].

\subsection{Evaluation Metrics}

In the paper, the successful forwarding rate represents the network resilience when network nodes fail, and the average path length shows the network performance with different routing methods. Therefore, we evaluate the successful forwarding rate and the average path length of different routing methods with two kinds of network models. 
The successful forwarding rate is the ratio of packets successfully arriving at the destination node to all packets sent between the source to destination, which evaluates the performance of routing algorithm. Here we compute the successful forwarding rate as

$$
\mathrm{SFR}=\frac{\mathrm{n}_{\mathrm{s}}}{\mathrm{n}_{\mathrm{a}}}
$$

which $n_{s}$ represents the number of packets which successfully arriving at the destination node and $n_{a}$ is the number of all packets sent.

The average path length is the average hops between origin and destination as

$$
\mathrm{APL}=\frac{\sum_{i=1}^{\mathrm{i}=\mathrm{N}} \sum_{j=1}^{\mathrm{j}=N} h(i, j)}{N(N-1)}
$$

where $h$ depicts as the hops for a source-destination pair in the routing matrix of the network and $N$ is the number of nodes in the network.

\subsection{Successful forwarding rate in ER network}
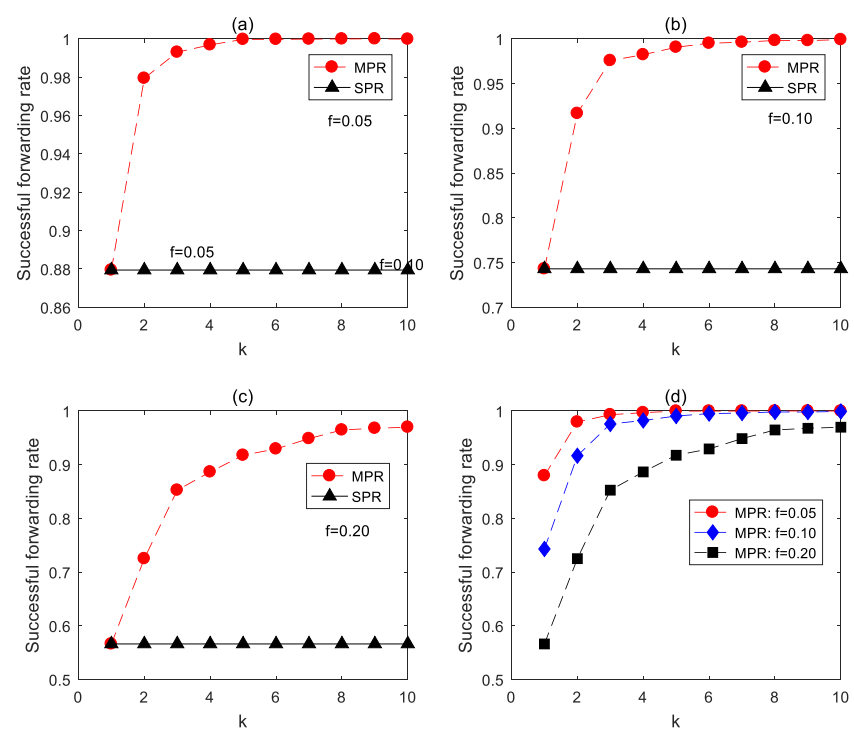

Fig. 3. The evolution of successful forwarding rate as a function of $k$ on the ER networks.

As we know, the successful forwarding rate of the network can evaluate the communication recovery efficiency of the network. Therefore, we test it with different 
numbers of backup paths $k$ and several failure rates $f$ as Fig.3 shows. In the Fig.3 (a), we investigate the comparisons of two routing algorithms(MPR and SPR) with different $k \in(1,10)$ and the same $f=0.05$ under ER network. In the ER networks, one can see that the MPR have greater successful forwarding rate than the SPR. And when $k$ is greater than 5, the success forwarding rate of MPR is equal $100 \%$ which mean all the packets will arrive their destinations. However, there is nearly no change in SPR.

In the Fig.3 (b), we increase the failure rate to 0.1 , and the rest of the parameters remains unchanged. We find that when the successful forwarding rate $f$ of MPR reaches $100 \%$, the number of backup path $k$ become 7 which is larger than the $k$ when $f=0.05$. The successful forwarding rate of SPR is reduced from $88 \%$ to $74 \%$.

In the Fig. 3 (c), we increase the $f$ again, the failure rate reaches $20 \%$ and the successful forwarding rate can reach $98 \%$ most. Meanwhile, SPR is reduced from $74 \%$ to $56 \%$. In general, the successful forwarding rate of MPR is higher than that of SPR. Moreover, when the node failure rate reaches $20 \%$, the success rate of MPR keeps a high value.

In the Fig.3(d), we can see that the MPR have good performance in different node failure rate. The higher node failure rate is, the more backup paths achieved. Our MPR routing appears to achieve high robustness against the node failure.

\subsection{Successful forwaring in BA network}
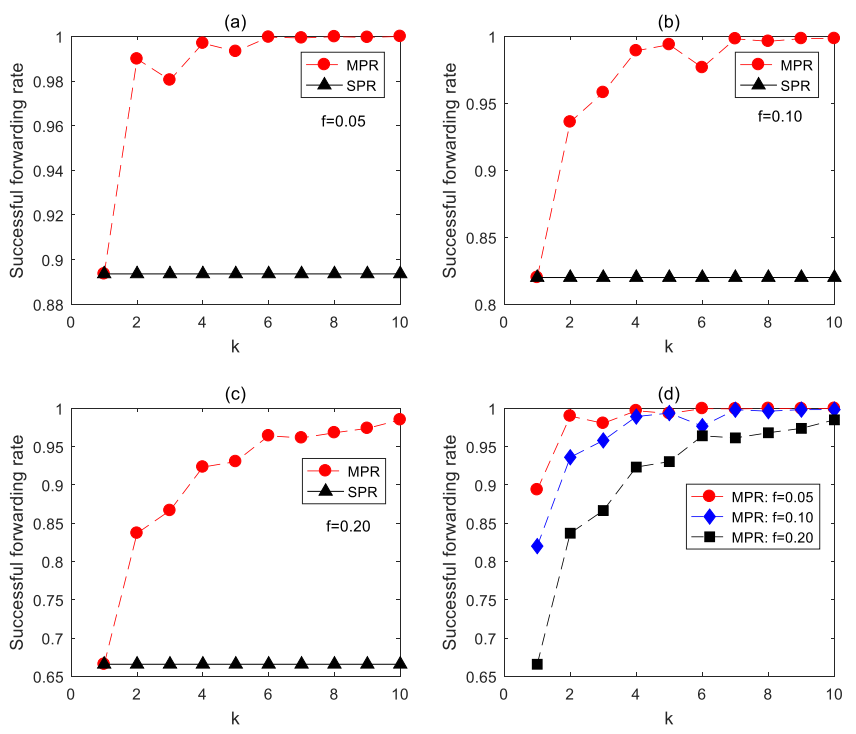

Fig. 4. The evolution of successful forwarding rate as a function of $k$ on the BA networks. 
In the Fig. 4 we set $k \in(1,10)$ and $f=0.05,0.1$ in BA networks and 0.2 and compare the successful forwarding rate of MPR and SPR. In the Fig.4 (a), we investigate the comparisons of the two routing algorithms with different $k \in(1,10)$ and the same $f=0.05$ under the BA network. In the BA networks, we can see that the MPR has better successful forwarding rate than the SPR. And when $k$ is greater than 6 and node failure rate is $5 \%$, all the packets will arrive successfully their destinations. However the successful forwarding rate is nearly no change in SPR which is equal to $89 \%$.

In the Fig.4 (b), we increase the failure rate to 0.1 , and the rest of the parameters remains unchanged. We find that when the successful forwarding rate $f$ of MPR reaches $100 \%$, the number of backup path $k$ become 7 which is larger than the $k$ when $f=0.05$. The successful forwarding rate of SPR is reduced from $89 \%$ to $82 \%$.

In the Fig.4 (c), we increase the $f$ again, the failure rate reaches $20 \%$ and the successful forwarding rate can only reach $98 \%$ most. However SPR is reduced from $82 \%$ to $66 \%$. In general, the successful forwarding rate of MPR is higher than that of SPR. And even when the node failure rate reaches $20 \%$, the success rate of MPR can reach $98 \%$.

In the Fig.4 (d), we can see that the MPR has good performance in different node failure rate. The higher node failure rate, the more backup paths.

\subsection{Comparisons of successful forwarding rate for BA network and ER network}
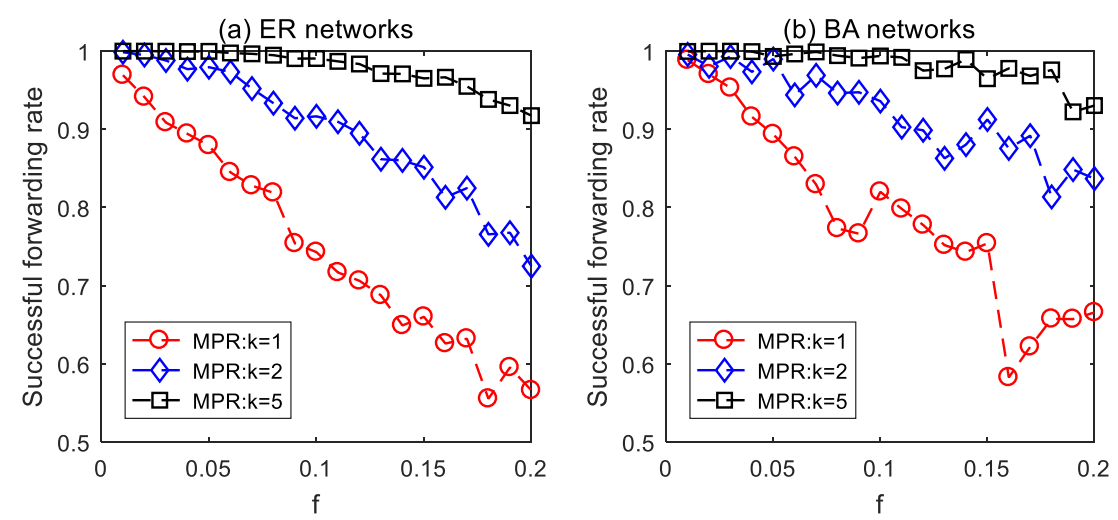

Fig. 5. The evolution of successful forwarding rate as a function of $f$ on the BA and ER network.

In the Fig.5(a)-(b), we can see the effect of $k$ value for the successful forwarding rate. The $k$ value is larger and the successful forwarding rate is larger. Especially with the $f$ is becoming larger, more nodes lost and the number of backup paths is more important. As the figure shows, when $k=5$, even the node failure rate reaches $20 \%$, we can restore more than $90 \%$ of communi-cations in the BA and ER networks.

\subsection{The average path length with different $k$ in ER and BA networks}


As we know, it is easy to increase the average path length while optimizing the routing algorithm. Therefore we test it with different number of backup paths $k$ in Fig.6. In the Fig.6 (a), we investigate the comparisons of three different values of $f$ with different $k \in(1,10)$ under ER network. In the ER network, with the value of $k$ is larger, and the average path length is smaller. Because larger $k$ means more feasible options for the alternative path switch. And when $k$ is equal 4 , the average path length just is nearly unchanged.

In the Fig.6 (b), the BA network is complicated, however the average path length tends to be stable and the value is lower than that in the ER network. As the Fig. 6 (b) shows, although there are some fluctuations, the average path length is becoming smaller as a whole with the $k$ is becoming larger. With higher failure rate, the available alternative path number reduces, and larger average path length is needed.

(a) ER network

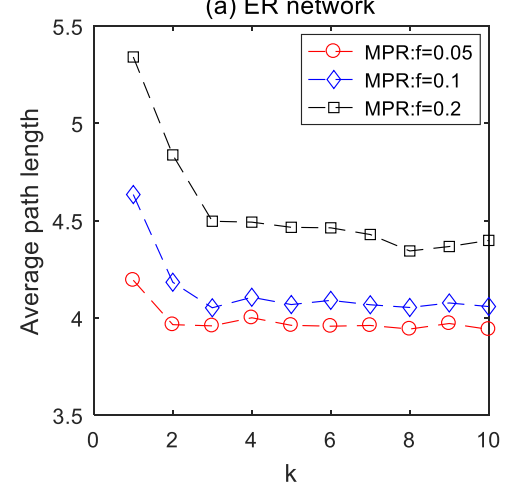

(b) BA network

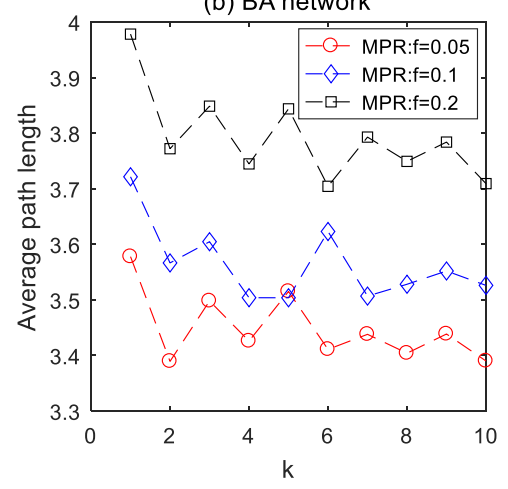

Fig. 6. The evolution of the average path length as a function of $k$ in ER and BA networks.

\subsection{The average path length with different $f$ in ER and BA networks}
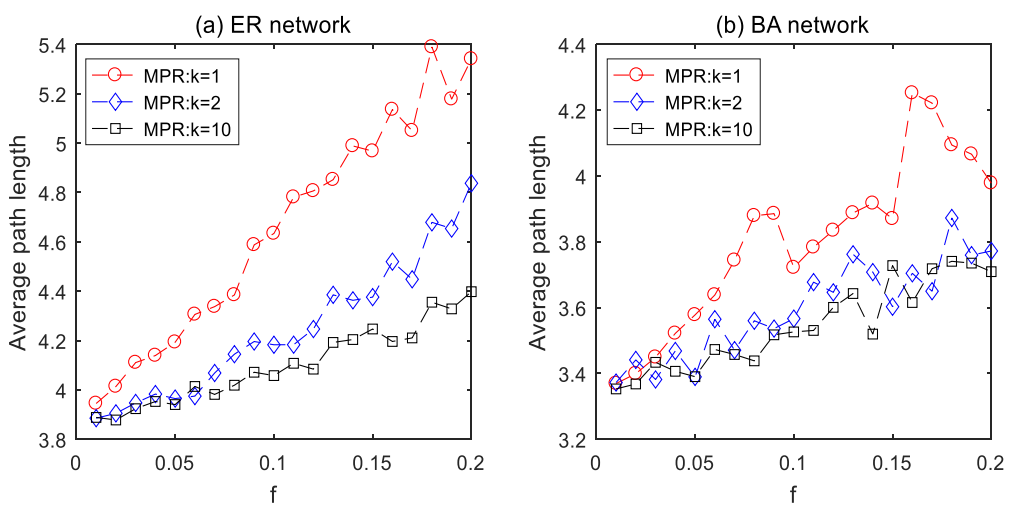

Fig. 7. The evolution of the average path length as a function of $f$ in ER and BA networks. 
In the Fig.7 (a), we investigate the comparisons of three different values of $f$ with different $k \in(1,2,10)$ in ER network. In the ER network, with the value of $f$ is larger, and the average path length is larger which varies from 3.9 to 5.3 because the $f$ is larger and there are more links disconnect. Therefore, packets arriving at the destination node will pass through more nodes. When the number of the backup path is larger, the average path length is shorter.

In the Fig.7 (b), the average path length of the BA network varies from 3.3 to 4 when the $f$ changes from 0.05 to 0.2 . However when $k=2$ and $k=10$, the average path length of the BA network has not been an obvious change with the same $f$.

\section{Conclusion}

To sum up, this work studied the resilient multi-path routing for the SDN based spacebased network. The multi-path routing increases the successful forwarding rate and reduces the average path length when the nodes failure occur. When the rate of nodes failure of SBN is high, the resilience of the network is still high when the number of backup paths is big enough. Therefore the multi-path routing is meaning for the resilience of a space-based network based on SDN, and has potential applications in the future generation of communication technology such as the NFV (Network Functions Virtualization) architecture [21-22].

\section{Acknowledgements}

This work was supported by the Natural Science Basis Research Plan in Shaanxi Province of China (2020JM-203), the research program of Science and Technology on Communication Networks Laboratory (6142104190306), and the National Natural Science Foundation of China (61502375).

\section{References}

[1] Richharia M. Mobile Satellite Communications: Principles and Trends[M]John Wiley \& Sons, Ltd, 2014: 37-87.

[2] Werner M. A dynamic routing concept for ATM-based satellite personal communication networks[J]. IEEE Journal on Selected Areas in Communications, 1997, 15(8): 1636-1648.

[3] Lu N, Zhang H, Ma J. A novel inter-satellite routing protocol based on link recognizing[C]. International Conference on Cyberspace Technology. IET, 2014: 1-4.

[4] Li C, Liu C, Jiang Z, et al. A Novel Routing Strategy Based on Fuzzy Theory for NGEO Satellite Networks[C]. Vehicular Technology Conference. IEEE, 2015: 1-5.

[5] Liu Z, Han J, Wang Y, et al. Performance analysis of routing algorithms in satellite network under node failure scenarios[C]. Global Communications Conference. IEEE, 2014: 2838-2843.

[6] Wang J, Qiao L, Shao S, et al. High-performance routing search algorithm in satellite IP switches[C]. International Conference on Computer Science and Network Technology. IEEE, 2014: 863-866.

[7] Liu Y, Feng W, Sun H, et al. An Algorithm Design of Inter-satellite Routing Based on Fibonacci Heap[C]. Fourth International Symposium on Computational Intelligence and Design. IEEE Computer Society, 2011: 51-54.

[8] Taleb T, Mashimo D, Jamalipour A, et al. SAT04-3: ELB: An Explicit Load Balancing Routing Protocol for Multi-Hop NGEO Satellite Constellations[C]. Global Telecommunications Conference, 2006. GLOBECOM '06. IEEE. IEEE, 2007: 1-5.

[9] Xu Z, Meng X, Cheng Z. The integrated routing algorithm for multi-satellites, multi-groundstations and multi-processing-centers[C]. International Conference on Natural Computation. IEEE, 2014: 1605-1609 
[10] Yan G, Zhou T, Hu B, et al. Efficient Routing on Complex Networks[J]. Physical Review E, 2006, 73(4):046108.

[11] Barabasi A L, and Albert R, Emergence of Scaling in Random Networks[J], Science, 1999,286(5439):509-512.

[12] Zhou J, Yu X, and Lu J. Node importance in controlled complex networks[J], IEEE Transactions on Circuits and Systems II: Express Briefs, 2019 , 66[3]:437-441.

[13] Wu J, You W, Wu T and et al, Abnormal phenomenon in robustness of complex networks with heterogeneous node functions[J], Physica A Statistical Mechaniscs\& Its Application, 2018, 506.

[14] Wu J, Zhong J, Chen Z and et al, Optimal coupling patterns in interconnected communication networks[J], IEEE Transactions on Circuits and Systems II: Express Briefs, 2018, 65(8):11091113.

[15] Chen G, Wen G, and Yu X, Performance analysis of distributed shortpath set based routing in complex networks[J], IEEE Transactions on Circuits and Systems II: Express Briefs, 2019, 66(8):1426-1430.

[16] Dijkstra E W, A note on two problems in connexion with graphs[J], Numerische Mathematik, 1959, 1(1): 269-271 .

[17] Gounder V V, Prakash R, Abu-Amara H. Routing in LEO-based satellite networks[C].Wireless Communications and Systems, Emerging Technologies Symposium. IEEE, 1999: 221-226

[18] Chang H S, Kim B W, Chang G L, et al. FSA-based link assignment and routing in low-earth orbit satellite networks[J]. IEEE Transactions on Vehicular Technology, 2002, 47(3): 10371048 .

[19] Albert R, Barabasi A L. Statistical mechanics of complex networks[J]. Review of Modern Physics, 2002,74(10):47-97.

[20] Barabasi A L, Albert R, Jeong H.Mean-field theory for scale-free random networks[J]. Physica A: Statistical Mechanics and its Applications, 1999, 272(1):173-187.

[21] Liu J, Jiang Z, Kato N, Akashi O, Takahara A, Reliability Evaluation for NFV Deployment of Future Mobile Broadband Networks[J], IEEE Wireless Communications, 2016, 23(3): 90-96.

[22] Jiang Z, Chen Z, Ma J, Zhang Y, Gu J, Traffic Dynamics Evaluation for the Future NFV Deployment[J], in IEEE Transactions on Circuits and Systems II: Express Briefs, 2019, Online ready, DOI: 10.1109/TCSII.2019.2962249. 